\title{
Balkanologie
}

Balkanologie Revue d'études pluridisciplinaires

Vol. IX, n' 1-2 | 2005

Volume IX Numéro 1-2

\section{Diasporas musulmanes balkaniques dans l'Union Européenne}

Introduction

Balkan Muslim Diasporas in the EU

Xavier Bougarel et Dimitrina Mihaylova

\section{OpenEdition}

\section{Journals}

Édition électronique

URL : http://journals.openedition.org/balkanologie/577

DOI : 10.4000/balkanologie. 577

ISSN : 1965-0582

\section{Éditeur}

Association française d'études sur les Balkans (Afebalk)

Édition imprimée

Date de publication : 1 décembre 2005

ISSN : 1279-7952

\section{Référence électronique}

Xavier Bougarel et Dimitrina Mihaylova, « Diasporas musulmanes balkaniques dans I'Union

Européenne », Balkanologie [En ligne], Vol. IX, n 1-2 | 2005, mis en ligne le 13 janvier 2010, consulté le

17 décembre 2020. URL : http://journals.openedition.org/balkanologie/577 ; DOI : https://doi.org/

10.4000/balkanologie.577

(c) Tous droits réservés 


\section{DOSSIER}

\section{DIASPORAS MUSULMANES} BALKANIQUES DANS L'UNION EUROPEENNE 


\title{
DIASPORAS MUSULMANES BALKANIOUES DANS L'UNION EUROPEENNE
}

\section{INTRODUCTION}

\author{
Xavier Bougarel, Dimitrina Mihaylova*
}

Ce dossier, consacré aux musulmans d'origine balkanique vivant au sein de l'Union européenne, est composé de six articles ${ }^{1}$. Ceux de Dimitris Antoniou, Jeanne Hersant, Kristina Grünenberg et Fabrizio Speziale ont d'abord été présentés en novembre 2003 , lors d'un colloque sur Les musulmans balkaniques et l'islam en Europe de l'Ouest, co-organisé par l'Association française d'études sur les Balkans (AFEBalk) et le laboratoire Migrinter du CNRS (UMR 6588, Poitiers) ${ }^{2}$. Une première version de l'article de Špela Kalčić a été présentée lors de la huitième conférence de l'Association européenne d'anthropologie sociale (EASA), tenue à Vienne en septembre 2004. Enfin, l'article de Georgia Kretsi a d'abord été publié en allemand, comme chapitre d'un ouvrage collectif intitulé Le vaste monde et le village. L'émigration albanaise à la fin du vingtième siècle 3 .

Cette diversité dans la provenance des articles rassemblés ici se double d'une relative variété dans leurs cadres théoriques et leurs problématiques concrètes. Ainsi, les auteurs insistent tous sur la nature multiple et fluctuante

\footnotetext{
- Xavier Bougarel est Chargé de recherche au CNRS, laboratoire " Etudes turques et ottomanes ", Paris. Contact : xbougarel@yahoo.fr ; Dimitrina Mihaylova est Research Fellow au Centre on Migration, Policy and Society (COMPAS), Oxford. Contact : dimitrina.mihaylova@linacre.oxford.ac.uk

${ }^{1}$ Nous tenons à remercier Nathalie Clayer, Mirjana Morokvašić, Pierre Sintès, Gilles de Rapper et Katerina Seraïdari pour l'aide qu'ils nous ont apportée dans la réalisation de ce dossier.

${ }^{2}$ Nous tenons à remercier le ministère de la Recherche pour avoir soutenu financièrement ce colloque, la Maison des Sciences de l'Homme et de la Societé de Poitiers pour l'avoir accueilli dans ses murs, et plus particulièrement Yves Tomić de l'Association française d'études sur les Balkans et Kamel Doraï du laboratoire Migrinter pour en avoir assuré dans une large mesure les dimensions administratives et logistiques.
}

${ }^{3}$ Kaser (Karl), Richler (Robert), Schwandner-Sievers (Stephanie), Hg, Die weite Welt und das Dorf. Albanische Emigration am Ende des 20. Jahrhunderts, Wien : Böhlau, 2002. 
des appartenances collectives, mais en allant plus ou moins loin dans leur déconstruction, et en utilisant malgré tout des concepts tels que celui d"'identité", ou en les évitant soigneusement 4 . De mème, tous s'inspirent de méthodes dérivées de l'anthropologie (observation participante, entretiens non-directifs, etc.), mais pour étudier des objets extrêmement variés.

Les recherches de Kristina Grünenberg et de Georgia Kretsi, par exemple, portent sur les pratiques privées d'individus souvent qualifiés de "musulmans sociologiques", à savoir des individus dont les origines familiales musulmanes ne se traduisent pas forcément par une identification active à la "communauté musulmane" ou par une religiosité particulière. Dimitris Antoniou, Jeanne Hersant et Špela Kalčić s'intéressent plutôt aux marqueurs identitaires et aux pratiques collectives qui structurent certains groupes, ainsi qu'aux acteurs associatifs qui cherchent indissociablement à en fixer les contours et à en garder le contrôle. Enfin, Fabrizio Speziale concentre son attention sur un maître soufi et le cercle de ses disciples, ainsi que sur l'évolution de leur identité religieuse. De telles variations dans les perspectives théoriques, les problematiques et les échelles choisies ne rendent pas vaine toute tentative de comparaison, bien au contraire, mais impliquent un examen attentif des catégories à travers lesquelles celle-ci peut s'effectuer.

\section{DES DIASPORAS D'ORIGINE RÉCENTE}

La diversité ethnolinguistique des populations musulmanes des Balkans, les clivages socioculturels et politiques qui les traversent, leurs rapports différenciés aux populations non-musulmanes et aux États de la région ont déjà fait l'objet de plusieurs ouvrages 5 , et il n'est pas utile d'y revenir dans le cadre de ce dossier. Il faut par contre noter que, quelque soit le sujet traité, les auteurs rassemblés ici soulignent tous que l'expérience migratoire, son impact sur les modes d'identification et de structuration du groupe, les pratiques et les solidarités religieuses, ne peuvent ètre analysés sans tenir compte des contextes et

4 Sur les problèmes liés à l'utilisation du concept d'"identitè" en sciences sociales, voir Brubaker (Rogers), Coopers (Frederick), "Beyond "Identity" ", Theory and Society, 29 (1), février 2000.

5 Voir entre autres Popovic (Alexandre), L'islam balkanique. Les musulmans du sud-est européen dans la période post-ottomane, Berlin-Wiesbaden : Otto Harrassowitz, 1986 ; Poulton (Hugh), Taji-Farouki (Suha), eds., Muslim Identity and the Balkan State, London : Hurst, 1997 ; Bougarel (Xavier), Clayer (Nathalie), éds., Le nouvel Islam balkanique. Les musulmans, acteurs du post-communisme (1990-2000), Paris : Maisonneuve \& Larose, 2001 ; Karčić (Fikret), Muslimani Balkana : istočno pitanje u XX. vijeku (Musulmans des Balkans : la Question d'Orient au XX ${ }^{\text {eme }}$ siècle), Tuzla : Behram-begova medresa, 2001. 
des pratiques antérieures à la migration, et des évolutions propres au pays d'origine : ainsi, Fabrizio Speziale voit dans la capacité d'adaptation à des environnements variables et hostiles une constante de l'identité tsigane, Georgia Kretsi situe les changements de prénom des migrants albanais dans le prolongement de la culture anthroponymique des Albanais musulmans et de la politique d'albanisation des prénoms, Kristina Grünenberg rappelle que la limitation de la religion à la sphère privée est aussi un legs du communisme yougoslave, Špela Kalčić lie les évolutions de l'identité bosniaque aux conséquences de l'indépendance slovène comme aux échos de la guerre en BosnieHerzégovine, Dimitris Antoniou et Jeanne Hersant montrent comment les populations et les associations qu'ils étudient sont influencées par le contexte plus général des relations gréco-turques.

Certes, à bien des égards, l'émigration constitue une rupture, d'autant plus lorsqu'elle prend la forme d'un exil forcé (Bosnie-Herzégovine, Kosovo) ou succède à plusieurs décennies de confinement territorial (Albanie, zone militaire grecque). Mais elle n'est jamais synonyme de tabula rasa, tant au niveau des pratiques culturelles que des réseaux sociaux ${ }^{6}$ et, pour ce qui nous intéresse plus particulièrement ici, elle ne met pas fin à la diversité des populations musulmanes balkaniques. Dès lors, rassembler sous l'intitulé Diasporas musulmanes balkaniques plusieurs articles consacrés aux Turcs, aux Albanais, aux Bosniaques et aux Roms des Balkans vivant en contexte diasporique ou "semidiasporique" (voir infra) ne peut se justifier que dans la mesure où ces différentes populations ont une expérience diasporique qui, tout à la fois, les rapproche les unes des autres et les distingue d'autres groupes originaires des pays balkaniques ou du monde musulman.

De ce point de vue, la première question qui se pose est de savoir si les musulmans d'origine balkanique vivant au sein de l'Union européenne constituent ou non des diasporas. Sans doute, ces dernières décennies ont-elles été marquées par une émigration massive des musulmans balkaniques vers l'Europe occidentale, et ce sont désormais plusieurs centaines de milliers de musulmans originaires des Balkans qui résident dans différents pays de l'UE, y représentant parfois une part importante de la population d'origine étrangère (Albanais d'Albanie en Grèce et en Italie, Bosniaques en Suède, en Allemagne et en Autriche, Albanais du Kosovo en Belgique, etc.). Dans les cas grec et yougoslave, l'émigration de Gastarbeiter musulmans vers l'Europe occidentale a commencé dans les années 1960, mais est restée limitée et a continué de se heurter à certains obstacles administratifs, comme le rappelle

6 Voir entre autres Sintès (Pierre), "Les Albanais en Grèce. Le rôle des réseaux préexistants ", Balkanologie, 7 (1), juin 2003 (dossier « Migrations et diasporas "). 
Jeanne Hersant à propos de la Thrace occidentale7. Il faut donc attendre les années 1990, avec la libre circulation entre la Grèce et les autres États de l'UE, et surtout la fin chaotique et violente des régimes communistes, pour que l'émigration des musulmans balkaniques prenne un caractère massif : en une décennie, 800 ooo Albanais - dont un nombre indéterminés de "musulmans sociologiques" 8 - cherchent par tous les moyens à fuir la misère et le chaos de l'Albanie post-communiste ${ }^{9}$, cependant que plusieurs centaines de milliers de réfugiés bosniaques et albanais kosovars se retrouvent chassés de leurs foyers par la guerre, la répression et le "nettoyage ethnique"10.

Cette émigration massive vers l'Europe occidentale constitue un tournant majeur par rapport à la première moitié du vingtième siècle, lorsque les flux migratoires des musulmans balkaniques étaient orientés vers Istanbul et l'Anatolie $^{11}$, et que les rares musulmans d'origine balkanique présents en Occident tendaient soit à se fondre au sein de communautés plus vastes et

7 Les populations musulmanes de Yougoslavie n'ont pas connu de restrictions spécifiques après la restauration de la liberté de circulation pour l'ensemble des citoyens yougoslaves en 1963, mais leur taux d'émigration est longtemps resté inférieur à celui des populations croate, serbe ou macédonienne. Ainsi, selon les résultats du recensement de 1981, sur les 875000 citoyens yougoslaves résidant à l'étranger, 63000 (7,2\%) étaient de nationalité albanaise et $58000(6,6 \%)$ étaient de nationalité musulmane (bosniaque), quand ces deux groupes représentaient respectivement $7,7 \%$ et $8,9 \%$ de la population yougoslave totale.

${ }^{8}$ Outre que le niveau de pratique religieuse des Albanais d'Albanie est particulièrement bas, il n'existe pas de données statistiques récentes sur l'appartenance confessionnelle de la population albanaise d'Albanie en général ou de la diaspora albanaise en particulier. Si l'on se réfère aux résultats du recensement de 1942 (le dernier à avoir pris en compte l'appartenance confessionnelle), d'après lequel deux tiers environ de la population albanaise était de confession musulmane, on peut émettre l'hypothèse qu'environ 500000 Albanais d'Albanie installés en Europe occidentale seraient de tradition musulmane.

9 Voir entre autres King (Russell), Schwandner-Sievers (Stephanie), Mai (Nicola), eds., The New Albanian Migration, Brighton : Sussex Academic Press, 2005 ; De Rapper (Gilles), "Transformation und Anpassung. Die albanischen Zuwanderer in Griechenland zwischen Integration und Rassismus ", in Brunnbauer (Ulf), Hg., Umstrittene Identitäten. Ethnizität und Nationalität in Südosteuropa, Frankfurt am Main : Peter Lang, 2002 ; Sintès (Pierre), art.cit.

10 Voir entre autres Albert (Sophie), Les réfugiés bosniaques en Europe, Paris : Montchrétien, 1995 ; Van Selm (Joanne), ed., Kosovo's Refugees in Europe, London : Pinter, 2000 ; Vatz-Laaroussi (Michèle), Manço (Altay), ed., Jeunesses, citoyennetés, violences : réfugiés albanais en Belgique et au Québec, Paris : L'Harmattan, 2003 ; Brochmann (Grete), "Bosnian Refugees in the Scandinavian Countries : A Comparative Perspective on Immigration Control in the 1990s n, New Community, 23 (4), octobre 1997.

${ }^{11}$ L'exode massif des Turcs de Bulgarie vers la Turquie en 1989 s'inscrit dans la continuité de ces flux migratoires mais, contrairement à ce qui s'était passé lors de précédentes vagues migratoires, la moitié d'entre eux retourneront en Bulgarie au cours de la décennie suivante. Voir Zhelyazkova (Antonina), ed., Between Adaptation and Nostalgia : The Bulgarian Turks in Turkey, Sofia : International Center for Minorty Research, 1998 ; Parla (Ayșe), " Marking Time along the Bulgarian-Turkish Border ", Ethnography, 4 (4), 2003 ; Krasztev (Petar), " Understated, Overexposed : Turks in Bulgaria, Immigrants in Turkey ", Balkanologie, 5 (1-2), décembre 2001. Sur l'émigration albanaise vers la Turquie dans les années 1990, voir De Rapper (Gilles), Les Albanais à Istanbul, Istanbul : IFEA, 2000. 
mieux structurées (turque, yougoslave, croate, etc.) ${ }^{12}$, soit à constituer des cercles restreints d'émigrés politiques ${ }^{13}$. Mais les populations musulmanes balkaniques désormais installées dans différents pays de l'UE peuvent-elles pour autant être considérées comme des diasporas ? Les situations variant considérablement d'une population à l'autre, la réponse ne peut être que nuancée. Ainsi, l'émigration des Albanais d'Albanie vers la Grèce reste souvent temporaire ou saisonnière, malgré plusieurs vagues de régularisation après 1997. Elle ne se traduit pas par l'affirmation d'une identité communautaire forte ou par la constitution d'un tissu associatif dense $\mathrm{e}^{14}$, mais par un désir d'assimilation et d'“invisibilité"15 dont les changements de prénom décrits par Georgia Kretsi ne constituent qu'un aspect. De telles pratiques se retrouvent chez les musulmans de Thrace occidentale installés à Athènes, et étudiés par Dimitris Antoniou. Dans le cas des Turcs de Thrace installés en Allemagne, au contraire, Jeanne Hersant montre comment les associations et, à travers elles, diverses pratiques sociales plus ou moins formalisées servent non seulement à préserver la cohésion du groupe en contexte migratoire, mais aussi à le mobiliser politiquement et à tirer profit de cette position excentrée pour agir sur le pays d'origine ${ }^{16}$. Ces processus correspondent bien aux critères généralement avancés pour juger de l'existence d'une diaspora, à savoir l'existence de

plusieurs des traits suivants : (1) une dispersion, souvent traumatisante, hors du pays d'origine ; (2) à défaut, un départ du pays d'origine à la recherche de travail, de débouchés commerciaux ou d'ambitions coloniales; (3) une mémoire et un mythe collectifs autour du pays d'origine ; (4) une idéalisation de la supposée terre

\footnotetext{
12 Voir Imamović (Mustafa), " Bosanskomuslimanska dijaspora u SAD " (La diaspora bosno-musulmane aux USA), in Šehic (Nusret), ur., Migracije i Bosna i Hercegovina (Les migrations et la Bosnie-Herzégovine), Sarajevo : Institut za istoriju, 199o, pp. 349-356 ; Blumi (Isa), « Defining Social Spaces by Way of Deletion : The Untold Story of Albanian Migration in the Postwar Period ", Journal of Ethnic and Migration Studies, 29 (6), novembre 2003 (dossier " Albanian Migration and New Transnationalisms "); Gjeloshaj (Kolë), " Qui sont les Albanais de Belgique? ", Agenda interculturel, (228), décembre 2004.

13 Sur l'émigration politique bosniaque, voir Imamović (Mustafa), Bošnjaci u emigraciji. Monografija "Bosanskih pogleda " 1955-1967 (Les Bosniaques dans l'émigration. Monographie de "Points de vue bosniens" 1955-1967), Sarajevo / Zürich : Bošnjački institut, 1996 ; Karić (Enes), Filandra (Šaćir), Bošnjačka ideja (L'idée bosniaque), Zagreb : Globus : 2002. Sur l'émigration politique albanaise, voir Lipsius (Stephan), " Untergrundorganisationen im Kosovo. Ein Überblick ", Südosteuropa, 47 (1-2), janvier-février 1998 ; Xhudo (Gus), " Albania's Émigrés : Helpful or Armful ? ", Jane's Intelligence Review, 8 (3), mars 1996.

14 Sur les associations de migrants albanais en Grèce, voir De Rapper (Gilles), art.cit. pp. 228-231.

15 Sur les " stratégies d'invisibilité " des migrants albanais en Grèce, voir Kokkali (Ifigenia), " Albanian Immigration and Urban Transformations in Greece - Albanian Migrant Strategies in Thessaloniki ", papier présenté à la conférence Current Social Science Research on Greece, London School of Economics, 10 juin 2005, accessible sur 〈http://www.lse.ac.uk/collections/hellenicObservatory .

${ }^{16}$ Voir également Hersant (Jeanne), " L'élaboration d'un discours identitaire dans l'espace migratoire des Turcs de Thrace occidentale ", Cahiers d'études dur la Méditerranée orientale et le monde turco-iranien, (34), juillet 2002 ; Hersant (Jeanne), « La minorité musulmane en Thrace occidentale et l'intégration européenne de la Grèce ", Études turques et ottomanes - documents de travail, (9-10), juin 2001.
} 
des ancêtres ; (5) un mouvement de retour ; (6) une conscience ethnique de groupe forte et durable ; (7) un rapport problématique avec les sociétés d'accueil ; (8) un sentiment de solidarité avec les membres du même groupe ethnique vivant dans d'autres pays ; (9) la possibilité d'une activité créatrice et enrichissante propre dans des pays d'accueil tolérants ${ }^{17}$.

De fait, les deux dernières décennies ont été marquées non seulement par la croissance rapide du nombre de musulmans d'origine balkanique vivant au sein de l'UE, mais aussi, dans bien des cas, par leur constitution en diasporas. L'insistance sur des identités nationales propres et sur les marqueurs qui leur sont associés, la création d'associations culturelles et leur fédération au niveau national, l'extension aux principaux pays d'accueil de l'activité des partis politiques apparus dans les Balkans dans les années 1990, l'investissement en retour du jeu politique des pays d'origine par d'anciennes générations d'émigrés politiques sont des phénomènes qui se retrouvent sous des formes variables chez les Bosniaques de Bosnie-Herzégovine et du Sandjak, les Albanais du Kosovo et de Macédoine, les Turcs de Thrace occidentale ${ }^{18}$. Cette structuration diasporique et ce développement de mobilisations politiques transnationales représentent un processus de rattrapage par rapport à d'autres diasporas balkaniques plus anciennement constituées, telles que les diasporas croate, serbe ou macédonienne ${ }^{19}$, et peut du reste impliquer une rupture avec des cadres associatifs plus larges, tels que les clubs encadrés par les consulats de Yougoslavie et destinés à tous les Gastarbeiter yougoslaves sans distinction de nationalité. Mais la mobilisation identitaire et politique des diasporas musulmanes balkaniques reflète avant tout des phénomènes similaires dans les pays d'origine, comme le rappellent Jeanne Hersant à propos des tensions croissantes que connaît la Thrace occidentale après 1974, ou Špela Kalčić à pro-

17 Cohen (Robin), Global Diasporas. An Introduction, Berkeley : University of California Press, 1997 , p. 180.

18 Voir entre autres Al-Ali (Nadje), "Trans- or A-National ? Bosnian Refugees in the UK and the Netherlands ", in Al-Ali (Nadje), Koser (Khalid), eds., New Approaches To Migration ? Transnational Communities and the Transformation of Home, London : Routledge, 2002 ; Eastmond (Marita), "Nationalist Discourses and the Construction of Difference : Bosnian Muslim Refugees in Sweden ", Journal of Refugee Studies, 11 (2), 1998 ; Wight (Ellen), Bosnians in Chicago : Transnational Activities and Obstacles to Transnationalism, Sussex : Center for Migration Research, 2000 ; Blumi (Isa), art.cit. ; Kostoricova (Denisa), Prestreshi (Albert), " Education, Gender and Religion : Identity Transformations among Kosovo Albanians in London ", Journal of Ethnic and Migration Studies, 29 (6), november 2003 ; Raganu (Nadège), "Recompositions identitaires et passage au politique des Albanais aux Etats-Unis", Balkanologie, 7 (1), juin 2003 (dossier " Migrations et diasporas ") ; Hersant (Jeanne), "L'èlaboration d'un discours identitaire " (art.cit.).

19 Voir entre autres Glamočak (Marina), Koncept Velike Hrvatske i Velike Srbije u političkoj emigraciji (Le concept de Grande Croatie et de Grande Serbie dans l'émigration politique), Užice : Kulturno-prosvetna zajednica Užice, 1997 ; Hockenos (Paul), Homeland Calling. Exile Patriotism and the Balkan Wars, Ithaca: Cornell University Press, 2003 ; Skrbiš (Zlatko), Long Distance Nationalism : Diasporas, Homelands and Identities, Aldershot : Ashgate, 1999 ; Danforth (Loring). The Macedonian Conflict : Ethnic Nationalism in a Transnational World, Princeton : Princeton University Press, 1995 ; Colera (Christophe), Une communauté dans un contexte de guerre : la " diaspora " serbe en Occident, Paris: L'Harmattan, 2003. 
pos de la montée des nationalismes en Bosnie-Herzégovine à la fin des années 1980.

Il convient enfin de mentionner certains contextes "semi-diasporiques", consécutifs aux migrations internes de musulmans d'origine rurale vers des centres urbains dépourvus de population musulmane autochtone. Un cas manifeste de situation "semi-diasporique" est celui des musulmans de Thrace occidentale installés à Athènes, dont Dimitris Antoniou explore les conditions de vie, les clivages identitaires et le tissu associatif. La communauté bosniaque en Slovénie étudiée par Špela Kalčić constitue un cas plus complexe de situation "semi-diasporique", dans la mesure où l'indépendance de la Slovénie et l'hostilité croissante envers les ressortissants des autres républiques de l'exYougoslavie en ont subitement modifié le statut juridique et symbolique. Or, ces populations vivant en contexte "semi-diasporique" ont elles aussi connu des évolutions identitaires et politiques significatives, comme l'attestent le cas de l'association Filotita auquel s'intéresse Dimitris Antoniou, ou la mobilisation des Bosniaques de Slovénie décrite par Špela Kalčić. De même, une étude sur les élites bosniaques installées en Croatie, leurs associations culturelles et - last but not least - la mosquée de Zagreb révèlerait sans doute que celles-ci ont joué dans les recompositions internes de leur communauté de référence un rôle aussi important que les organisations politico-militaires de la diaspora albanaise $^{20}$.

\section{L'« ISLAM EUROPÉEN 》 COMME DÉNOMINATEUR COMMUN ?}

Une fois établie l'existence de diasporas musulmanes balkaniques au sein de l'Union européenne, reste à déterminer si ces dernières, constituées sur une base ethno-nationale, partagent une expérience diasporique commune : en d'autres termes, qu'y a-t-il de commun entre les animateurs de l'association Filotita à Athènes, les familles turques de Thrace occidentale participant aux aile gecesi (soirées familiales), les migrants albanais se convertissant à l'orthodoxie, les jeunes salafistes de Jesenice rejetant l'islam "traditionnel" de leurs parents, les réfugiés bosniaques se conformant scrupuleusement aux normes de la société danoise, et les disciples roms de Shaykh Baba Jevat ?

Contrairement à ce que pourraient laisser croire certains discours sur les

\footnotetext{
20 Sur la communauté bosniaque en Croatie, voir Omerbašić (Ševko), Islam i Muslimani u Hrvatskoj (L'islam et les Musulmans en Croatie), Zagreb : Mešihat Islamske Zajednice u Hrvatskoj, 1999 ; ČičakChand (Ružica), "Islam i muslimani u Hrvatskoj : skica stvaranja muslimanskog/bos̄njačkog sociokulturnog prostora " (L'islam et les musulmans en Croatie : une esquisse de la formation d'un espace socioculturel musulman/bosniaque), Migracijske teme, 15 (4), 1999.
} 
"guerres balkaniques" ou sur la "forteresse Europe", ce ne sont ni les causes, ni les modalités concrètes de leur émigration : chassés par la guerre, victimes de la répression ou en quête de meilleures conditions de vie, certains musulmans originaires des Balkans vivent dans la clandestinité, d'autres bénéficient (pour combien de temps ?) du statut de réfugiés, les troisièmes ont obtenu un permis de séjour ou la citoyenneté du pays d'accueil. Ces trajectoires migratoires complexes et diversifiées ne sont en aucun cas propres aux diasporas musulmanes balkaniques. En outre, les articles rassemblés ici insistent tous sur les clivages socio-économiques, culturels, de genre et de génération qui traversent les populations étudiées, et qui se reflètent dans des rapports variables aux sociétés d'accueil, des capacités d'adaptation et des stratégies d'insertion différenciées ${ }^{21}$.

Que reste-t-il dès lors comme dénominateur commun, sinon l'identité religieuse des populations et des acteurs traités ici ? L'origine de ce dossier - un colloque sur Les musulmans balkaniques et l'islam en Europe de l'ouest -, tout comme son intitulé, ramènent inévitablement à cette question. Mais, pour éviter tout raisonnement tautologique, et toute essentialisation d'une hypothétique "identité musulmane balkanique", il faut insister d'emblée sur les différences de religiosité existant d'un groupe à l'autre et d'un individu à l'autre, sur la diversité des croyances et des pratiques religieuses, sur les multiples usages communautaires ou privés de la religion que révèlent les articles présentés ici.

$\mathrm{Au}$ demeurant, le fait que la plupart de ces articles ne soient pas centrés sur les questions religieuses permet de relativiser l'importance de la religion, dans la constitution des diasporas musulmanes balkaniques comme dans la vie quotidienne de leurs membres. Certes, Jeanne Hersant, Kristina Grünenberg, Špela Kalčić et Fabrizio Speziale montrent comment la commémoration des principales fêtes du calendrier islamique et de certains rites de passage, l'ouverture de lieux de prière, le respect de certains préceptes religieux contribuent à la cohésion du groupe. Un constat similaire se retrouve dans les travaux de Marita Eastmond ou de Nadja Al-Ali sur les réfugiés bosniaques, et de Denisa Kostovicova et Albert Prestreshi sur les réfugiés kosovars $^{22}$, et explique par exemple que le nombre de jama'ats (communautés locales de croyants) bosniaques en Europe occidentale soit passé de $3^{6}$ en avil

${ }^{21}$ Voir en particulier Al-Ali (Nadje), art.cit. ; Kostovicova (Denisa), Prestreshi (Albert), art.cit.

${ }^{22}$ Eastmond (Marita), art.cit. ; Al-Ali (Madje), art.cit. ; Kostovicova (Denisa), Prestreshi (Albert), art.cit. 
$1992^{23}$ à 136 dix ans plus tard ${ }^{24}$. Mais les migrants albanais d'Albanie constituent là encore une exception à la règle, pour les raisons indiquées par Georgia Kretsi : articulation spécifique entre identités religieuses et identité nationale albanaise depuis la fin du XIXème siècle, interdiction de toute activité religieuse entre 1967 et 1990 , pressions assimilatrices de la société grecque ${ }^{25}$. De façon plus générale, l'utilisation de la religion à des fins identitaires n'est jamais exclusive - la transmission de la langue maternelle ayant une importance au moins égale ${ }^{26}$-, et ne facilite en aucun cas le dépassement des clivages ethniques : les jama'ats yougoslaves créés en Allemagne dans les années 1980 se sont au contraire scindés en jama'ats bosniaques et albanais, et les ordres soufis implantés en Italie au sein de la diaspora rom en reproduisent les lignes de fracture ethniques et régionales, comme le note Fabrizio Speziale.

Par ailleurs, l'utilisation de certains symboles ou rituels religieux comme marqueurs identitaires ne signifie pas que le niveau de pratique religieuse soit élevé ou uniforme au sein des diasporas musulmanes balkaniques : au contraire, l'ensemble des auteurs - et de leurs interlocuteurs - souligne que la pratique régulière de la religion musulmane ne concerne qu'une minorité de croyants, composée avant tout de femmes, de personnes âgées et de familles d'origine rurale ${ }^{27}$. Ce décalage entre l'islam comme référent identitaire et l'islam comme système de croyance explique que, selon les centres d'intérêt et les approches de chaque auteur, des configurations similaires puissent susciter chez le lecteur des impressions, voire des conclusions sensiblement différentes, comme l'illustrent en particulier les articles de Kristina Grünenberg et Špela Kalčić. Dans la réalité, cependant, le "regard" slovène n'est sans doute pas

\footnotetext{
23 Mahmutovic (Mirsad), Dżemati Bošnjaka u dijaspori (Les jama'ats des Bosniaques de la diaspora). Sarajevo : Rijaset Islamske Zajednice, 2003. Voir également Klanco (Mustafa), "Muslimanske zajednice Bošnjaka u zapadnoj Evropi sa posebnim osvrtom na Njemačku " (Les communautés musulmanes de Bosniaques en Europe occidentale, et plus particulièrement en Allemagne), in Islamski centar, Islam u zapadnom svijetu (L'Islam dans le monde occidental), Zagreb : Islamski centar, 1997.
}

24 Mahmutović (Mirsad), op.cit. Sur le cas des jama'ats albanais de Belgique, voir Sula (Selvi), "Où prient les musulmans albanais? ", Agenda interculturel, (228), decembre 2004 .

25 Voir également Clayer (Nathalie), Religion et nation chez les Albanais XIXèmé-XXème siècles, Istanbul : Isis, 2003 ; De Rapper (Gilles), art.cit.

${ }^{26}$ L'éclatement de la fédération yougoslave et la "disparition" de la langue serbo-croate ont en particulier conduit à des demandes d'enseignement sèparé de la langue maternelle auprès des autorités des pays d'accueil. L'Autriche a ainsi mis en place des cours séparés de langue bosnienne dès le milieu des années 1990, et la Suède a reconnu à la fin des années 1990 l'existence séparée des langues serbe, croate et bosnienne, tout en maintenant un enseignement commun. Sur la question de l'enseignement de Ia langue maternelle en contexte diasporique, voir en particulier Eastmond (Marita), art.cit. ; Kostovicova (Denisa), Prestreshi (Albert), art.cit.

27 Voir également Al-Ali (Nadje), "Gender Relations, Transnational Ties and Rituals among Bosnian Refugees n, Global Networks, 2 (3), 2002 ; Collc-Peisker (Val), "At Least You're the Right Colour": Identity and Social Inclusion of Bosnian Refugees in Australia n, Journal of Ethnic and Migration Studies, 31 (4), July 2005 ; Sula (Selvi), L'islam et la communauté kosovare de Belgique, mémoire en vue de l'obtention du Diplôme approfondi en sciences politique, Université libre de Bruxelles, 2004- 
moins normatif que le "regard" danois, et la volonté d'intégration des Bosniaques pas moins grande en Slovénie qu'au Danemark. De même, la Communauté islamique de Bosnie-Herzégovine signale que 32 clubs culturels et 12 jama'ats bosniaques ont été créés au Danemark au cours des années $1990^{28}$, et plusieurs arrestations effectuées à Sarajevo et au Danemark en 2005 suggèrent qu'en Scandinavie également, de jeunes Bosniaques se sont laissés tenter par les sirènes de l'islam radical ${ }^{29}$.

La communauté de religion ne permet pas aux diasporas musulmanes balkaniques de dépasser leurs clivages ethniques ou nationaux et recouvre, en outre, une grande diversité de pratiques. La religion musulmane ne fonde donc pas, à elle seule, une communauté d'expérience entre musulmans d'origine balkanique. Nombre d'entre eux insistent par contre sur le fait qu'ils sont tout a la fois Européens et musulmans, que leur islam est un "islam européen", largement sécularisé et donc facilement adaptable aux normes des sociétés d'accueil. Cette idée d'un "islam européen" propre aux musulmans balkaniques se retrouve en particulier chez certains interlocuteurs de Kristina Grünenberg et Špela Kalčić, mais aussi, de manière plus implicite, chez les acteurs associatifs étudiés par Dimitris Antoniou et Jeanne Hersant ${ }^{30}$. Dans les faits, elle semble corroborée par les rapports distants, voire tendus, qui existent entre les musulmans d'origine balkanique et les autres populations musulmanes de l'UE. Kristina Grünenberg montre ainsi comment les réfugiés bosniaques cherchent à se démarquer des autres groupes de réfugiés et de migrants musulmans, Fabrizio Speziale décrit les conflits de doctrine entre Baba Jevat et les imams arabes de Florence ${ }^{31}$, Dimitris Antoniou évoque les rapports complexes existant à Athènes entre musulmans de Thrace occidentale et musulmans étrangers, et même les Turcs de Thrace occidentale étudiés par Jeanne Hersant ne manquent pas, à l'occasion, de dénoncer l'“arriération" des Turcs de Turquie.

Pourtant, insister sur les spécificités de l'islam balkanique et en faire ce qui, tout à la fois, rapprocherait les diasporas musulmanes balkaniques et les différencierait d'autres populations originaires des Balkans ou du monde musulman, soulève d'autres problèmes. Ainsi, le décalage entre islam comme référent identitaire et islam comme système de croyance se retrouve dans les usages que les diasporas grecque, serbe et croate font de l'orthodoxie ou du catholicisme, et dans le rapport que bien des populations musulmanes installées en Europe occidentale entretiennent à l'islam. L'idée selon laquelle les musul-

\footnotetext{
${ }^{28}$ Mahunutović (Mirsad), op.cit., pp. 85-105.

29 "Police Raid Raises Fears of Bosnia as Haven for Terrorists ", The New York Times, 04/12/05.

$3^{\circ}$ Voir également Al-Ali (Nadje), " Gender Relations, Transnational Ties and Rituals " (art.cit.) ; ColicPeisker (Val), art.cit.
}

${ }^{31}$ Sur les tensions entre croyants albanais et imams arabes, voir Kostovicova (Denisa), Prestreshi (Albert), art.cit., p. 1091. 
mans d'origine non-européenne seraient beaucoup plus pratiquants que ceux d'origine balkanique ne repose sur aucune étude sérieuse et, dans les faits, les écarts de religiosité entre diasporas musulmanes balkaniques ou en leur sein même - entre migrants d'origine rurale ou urbaine, entre première et deuxième générations, etc. - sont probablement plus importants ${ }^{32}$. Enfin, l'opposition entre un "islam européen" sécularisé ou hétérodoxe d'une part, un islam "non-européen" rigoriste d'autre part, est pour le moins simpliste : les confréries soufies sont plus actives au sein de certaines populations d'origine africaine ou asiatique que chez les musulmans d'origine balkanique ${ }^{33}$, et l'adaptation de la conscience et des pratiques religieuses au contexte diasporique est un phénomène général ${ }^{34}$.

Les différents articles présentés ici montrent du reste que les rapports entre les musulmans d'origine balkanique et ceux d'origine non-européenne ne sont ni univoques, ni immuables : Fabrizio Speziale suit de près les accommodations réciproques entre Baba Jevat et les imams arabes de Florence, Dimitris Antoniou signale que les animateurs de Filotita fréquentent les mêmes salles de prière que les musulmans étrangers (ce que refusent de faire d'autres musulmans de Thrace), et Fabrizio Speziale, Špela Kalčić et Jeanne Hersant notent que des acteurs religieux extérieurs - qualifiés selon les cas de "réformistes", "salafistes" ou "fondamentalistes" 35 - parviennent à recruter

$3^{2}$ En 1995, $14 \%$ seulement des musulmans français de la seconde génération déclaraient accomplir régulièrement leur prière (Dassetto (Felice), Marechal (Brigitte), Nielsen (Jorgen), éds., Convergences musulmanes. Aspects contemporains de l'islam dans l'Europe élargie, Paris : L'Harmattan, 2001, p. 27). Quelques années auparavant, $14 \%$ des jeunes Musulmans/Bosniaques de Bosnie-Herzégovine, $13 \%$ des jeunes Albanais du Kosovo et $50 \%$ des jeunes Albanais hors Kosovo affirmaient se rendre à la mosquèe pour des raisons religieuses (voir Pantic (Dragomir), "Prostome, vremenske i socijalne koordinate religioznosti mladih u Jugoslaviji " (Facteurs spatiaux, temporels et sociaux de la religiosité des jeunes en Yougoslavie), in Mihailovic (Srećko), ur., Deca krize. Omladina Jugoslavije krajem osamdesetih (Les enfants de la crise. La jeunesse de Yougoslavie à la fin des années 1980), Belgrade: IDN, 1990, p. 222\}.

33 Sur les confrèries soufies en général, voir Popovic (Alexandre), Veinstein (Gilles), éds., Les Voies d'Allah. Les ordres mystiques dans le monde musulman des origines à aujourd'hui, Paris: Fayard, 1996. Sur les confréries soufies en Europe occidentale, voir en particulier les chapitres de Jorgen Nielsen et Loïc Le Pape dans Allievi (Stefano), Nielsen (Jorgen), eds, Muslim Networks and Transnational Communities in and accross Europe, Leiden : Brill, 2003, ainsi que les articles de Benjamin Soares, Bruno Riccio et Kathryn Spellman dans Journal of Ethnic and Migration Studies, 30 (5), septembre 2004 (dossier « Islam, Transnationalism and Public Sphere in Western Europe ").

34 Voir entre autres Dassetto (Felice), Marechal (Brigitte), Nielsen (Jorgen), éds, op.cit. ; Dassetto (Felice). éd., Paroles d'Islam : individus, sociétés et discours dans llislam européen contemporain, Paris : Maisonneuve \& Larose, 2000 ; Roy (Olivier), Vers un Islam européen, Paris : Esprit, 1999 ; Nielsen (Jorgen), Towards a European Islam, Basingstoke : Macmillan, 1999.

35 Sur l'origine de ces termes et les problèmes liès à leur utilisation, voir Roy (Olivier), Gènéalogie de l'isslamisme, Paris : Hachette, 1995. 
une minorité de partisans au sein des diasporas musulmanes balkaniques, et exacerbent ainsi certains clivages internes ${ }^{36}$. Or, ce phénomène est non seulement commun à toutes les populations musulmanes de l'UE, mais s'inscrit dans un processus plus large de "globalisation de l'islam"37 : après tout, les jeunes salafistes décrits par Špela Kalčić ont d'abord été influencés par un Bosniaque ayant combattu en Bosnie-Herzégovine aux côtés des moujahiddins $^{38}$, et la Khalwatiyya à laquelle appartient sheykh Baba Jevat est ellemême une confrérie soufie de nature transnationale et en évolution constante ${ }^{39}$. Dès lors, si la revendication d'un "islam européen" peut servir de révélateur à une expérience diasporique propre aux musulmans d'origine balkanique, c'est à la condition de ne pas essentialiser cet islam, de ne pas en faire une donnée culturelle immuable et uniformément partagée.

\section{L'« INVISIBILITÉ » DES MUSULMANS D'ORIGINE BALKANIQUE}

Dans les articles rassemblés ici, deux thèmes reviennent comme des leitmotivs : l'alcool d'une part, le 11 septembre 2001 d'autre part $4^{\circ}$. Comme l'indique Kristina Grünenberg, la consommation d'alcool - réputée plus fréquente chez les musulmans d'origine balkanique - a aussi une forte dimension symbolique : Hajro boit ostensiblement une bière pour rasséréner ses nouveaux collègues de travail et, pour Armela et Mirzet, cette même boisson ne constitue pas seulement un plaisir gustatif, mais un " marqueur de similarité ou d'égalité avec les Danois ». De même, le fait que les attentats du 11 septembre aient

${ }^{36}$ Le conflit décrit par Špela Kalčić au sein de la communauté bosniaque en Slovénie se retrouve dans d'autres pays occidentaux, et s'est soldé par la création de jama'ats "dissidents" (Tevhid à Vienne, Tevhid à Munich, Behar à Grand Rapids, aux États-Unis, etc.) et d'organisations de jeunesse sans liens directs avec les principales associations culturelles bosniaques ou la Communauté islamique de BosnieHerzégovine (Jeunesse islamique active - Aktivna Islamska Omladina - en Autriche et en Allemagne, Union de la jeunesse islamique émigrée - Islamski Muhadžirski Omladinski Savez - en Suisse, Association des Musulmans d'Amérique du nord - Udruženje Muslimana Sjeverne Amerike - aux États-Unis, etc.).

37 Voir entre autres Ahmed (Akbar), Donnan (Hastings), eds., Islam, Globalization and Postmodernity, London : Routledge, 1994 ; Roy (Olivier), L'islam mondialisé, Paris : Seuil, 2002 ; Grillo (Ralph), " Islam and Transnationalism ", Journal of Ethnic and Migration Studies, 30 (5), septembre 2004, (dossier " Islam, Transnationalism and Public Sphere in Western Europe ").

${ }^{38}$ Sur la présence des moujahiddins en Bosnie-Herzégovine, voir Bellion-Jourdan (Jérôme), « Les réseaux transnationaux islamiques en Bosnie-Herzégovine ", in Bougarel (Xavier), Clayer (Nathalie), éds., op.cit.

39 Clayer (Nathalie), Mystiques, État et société. Les Halvetis dans l'aire balkanique de la fin du XVéme siècle à nos jours, Leiden : Brill, 1994 ; Clayer (Nathalie), "La Khalwatiyya (Khalvetiye) ", in Popovic (Alexandre), Veinstein (Gilles), (eds), op.cit.

$4^{\circ}$ Ces thèmes de l'alcool et du 11 septembre 2001 se retrouvent également chez Nadje Al-Ali, Val ColicPeisker, Denisa Kostovicova et Albert Prestreshi. 
conduit les musulmans d'origine balkanique à prendre encore d'avantage leurs distances envers leurs coreligionnaires d'origine non-européenne et à dénoncer comme "étrangères" les formes radicales de l'islam, ne s'explique pas uniquement par l'émotion ressentie ce jour-là : dans le cas de la communauté bosniaque en Slovénie, par exemple, Špela Kalčić estime qu'il s'agit aussi de se démarquer de pratiques que les croyants traditionnels " associent avec les cultures non-européennes des pays musulmans et, en tant que telles, avec quelque chose pouvant menacer leur position, en tant que Bosniaques et en tant qu'Européens ".

Au-delà du caractère "anecdotique" que peuvent revêtir ces deux thèmes (le taux d'alcool dans le sang n'a jamais constitué un indicateur fiable d'intégration sociale, et les sociétés européennes n'ont pas attendu le 11 septembre 2001 pour être islamophobes), leur récurrence montre que la question de l'“islam européen" revendiqué par les musulmans d'origine balkanique ne peut être considérée en dehors de son contexte, à savoir le statut de l'islam et des populations musulmanes dans leurs pays d'origine et dans leurs pays d'accueil. Ainsi, les modes de (non-)consommation d'alcool semblent indiquer la manière dont les musulmans d'origine balkanique négocient au jour au jour leurs rapports avec la société d'accueil et avec les autres populations musulmanes - ainsi que, bien souvent, leurs propres clivages internes -, et les attentats du 11 septembre semblent constituer un tournant dans la manière dont les musulmans d'origine balkanique sont perçus par leurs sociétés d'accueil, perçoivent ces sociétés et, in fine, se perçoivent et se définissent eux-mêmes.

La question se pose, dès lors, de savoir dans quelle mesure la revendication d'un "islam européen" par les musulmans d'origine balkanique est le reflet d'une socialisation religieuse spécifique antérieure à la migration, ou le produit dérivé d'une stratégie d'insertion dans les sociétés d'accueil. La réponse, on s'en doute, n'est pas univoque. Le fait d'être issus de sociétés pluriconfessionnelles et - à l'exception de la Grèce - post-communistes influence inévitablement la manière dont les musulmans d'origine balkanique se positionnent dans les sociétés d'accueil. Un siècle et demi d'expérience minoritaire et un demi-siècle de sécularisation autoritaire expliquent que la réduction de l'islam au statut de religion minoritaire et privée n'exige pas de leur part les mêmes ajustements que pour des musulmans originaires de pays majoritairement musulmans ${ }^{41}$. Mais les vertus explicatives de cette différence de socialisation religieuse restent limitées : après tout, les Turcs de Thrace occidentale étudiés par Jeanne Hersant déplorent que la société turque s'éloigne du modèle kéma-

41 Je remercie Samim Akgönül pour avoir attiré une première fois attiré mon attention sur ce fait en novembre 2003, lors du colloque sur Les musulmans balkaniques et l'islam en Europe de l'Ouest. Sur la question de l'expérience minoritaire propre aux Musulmans balkaniques, voir entre autres Popovic (Alexandre), op.cit.; Bougarel (Xavier), The Role of Balkan Muslims in Building a European Islam, Brussels : King Baudouin Foundation / European Policy Center, 2005, accessible sur 〈http://www.kbs-frb.be〉. 
liste, mais dépendent de leurs muftis pour régler leurs propres affaires familiales. Plus généralement, les pays musulmans n'ont pas - quoi qu'on en dise échappé aux processus de sécularisation, et l'opposition entre socialisation religieuse en contexte majoritaire ou minoritaire ne fait sens que pour la première génération de migrants.

Aux spécificités de la socialisation religieuse dans les pays d'origine s'ajoutent donc d'autres différences dans la manière dont les différentes populations musulmanes sont perçues et se perçoivent dans les pays d'accueil. Comme le montre Kristina Grünenberg, les Bosniaques occupent une place relativement privilégiée au sein de la hiérarchie symbolique des étrangers en vigueur dans la société danoise, en tant que "musulmans européens" d'une part, en tant que "vrais réfugiés" d'autre part. Un tel "privilège", toutefois, leur impose de se conformer strictement aux normes de cette même société. Cette position particulière des musulmans d'origine balkanique se retrouve dans d'autres contextes, quoi que sous des formes différentes. Ainsi, les migrants albanais étudiés par Georgia Kretsi sont loin de bénéficier dans la société grecque du statut symbolique d"'Autre légitime" ou de "quasi-Même" ; toutefois, contrairement aux autres musulmans étrangers, ils peuvent y accéder par le biais du changement de prénom et de la conversion à l'orthodoxie ${ }^{42}$. La revendication d'un "islam européen" par les musulmans d'origine balkanique, et l'effort d'adaptabilité et d'invisibilité religieuses qui l'accompagne, s'explique donc aussi par le fait que les sociétés d'accueil leur reconnaissent au moins indirectement un droit qu'elles refusent en général aux autres musulmans : celui de se considérer comme Européens.

Cette manière qu'ont les musulmans d'origine balkanique d'être perçus et de se percevoir simultanément comme musulmans et comme Européens reste précaire. D'une part, le fait de se voir reconnaître une identité européenne ne les protège pas de tout préjugé : les communautés albanaise et rom, par exemple, sont souvent associées à la petite délinquance et à la criminalité organisée43. D'autre part, tout évènement ravivant l'idée d'une incompatibilité radicale entre l'Europe et l'islam menace cette notion d'"islam européen" sur laquelle reposent - entre autres - les stratégies d'insertion d'une majorité de musulmans d'origine balkanique. Ceux-ci sont donc dans une position bien plus délicate que d'autres diasporas originaires des Balkans, pour lesquelles la mise en avant de marqueurs identitaires d'origine religieuse ne menace en

42 Voir également De Rapper (Gilles), art.cit. ; Kolkali (Ifigenia), art.cit.

$43 \mathrm{Su}$ la perception des Roms en Europe occidentale et orientale, voir Cambridge Review of International Affairs, 13 (2), printemps 2000 (dossier " Romani Migrations : Strangers in Anybody's Land ? ") ; Hommes \& Migrations, (1188-1189), juin-juillet 1995 (dossier " Tsiganes et voyageurs : entre précarité et ostracisme n). Sur l'évolution de la perception des Albanais en Italie, voir Zinn (Dorothy), "Adriatic Brethren or Black Sheep : Migration in Italy and the Albanian Crisis, 1991 ", European Urban and Regional Studies, 3 (3), 1996. 
rien leur identité européenne. C'est également dans ce contexte qu'il convient de resituer les changements de prénoms décrits par Georgia Kretsi et Dimitris Antoniou, les conversions à l'orthodoxie parmi les Albanais d'Albanie installés en Grèce ou, plus marginalement, les conversions au catholicisme ou au protestantisme parmi les Roms des Balkans et les Albanais du Kosovo44.

Lorsque les marqueurs identitaires d'origine religieuse ont joué un rôle essentiel dans les mobilisations politiques des années 1990, et ne peuvent ètre abandonnés sans fragiliser l'identité nationale, les phénomènes de conversion sont pratiquement inexistants, mais le recours à ces marqueurs identitaires reste confiné aux espaces privés ou communautaires. Travaillant sur la diaspora bosniaque en Australie, Val Colic-Peisker note par exemple que " seule une petite minorité de femmes musulmanes bosniennes d'origine rurale portent l'habit traditionnel avec un foulard qui couvre leurs cheveux, ce qui les rend visibles dans les suburbs australiens. (...) Dans le contexte australien, l'identité bosniaque (musulmane bosnienne) a plus une signification "interne" qu'“externe" : elle est un moyen de différenciation de ce groupe envers les deux autres groupes ethniques bosniens, les Serbes et les Croates, mais elle reste discrète et largement invisible "de l'extérieur", dans le contexte général australien $» 45$. Un constat similaire pourrait sans doute s'appliquer à la diaspora bosniaque en Amérique du nord et en Europe occidentale, à la nuance près que l'affichage de certains marqueurs religieux peut aussi relever de ruptures identitaires récentes et délibérées, comme l'atteste le cas des jeunes salafistes étudiés par Śpela Kalčic ${ }^{46}$.

Plus que le niveau de pratique religieuse, cette "invisibilité religieuse" des diasporas musulmanes balkaniques dans l'espace public est sans doute ce qui les différencie le plus des autres populations musulmanes vivant au sein de l'Union européenne. À partir de la fin des années 1970, en effet, la visibilité de l'islam s'est accrue en Europe occidentale, et certains enjeux symboliques forts tels que l'affaire Rushdie en Grande-Bretagne ou le port du voile en France ont servi de catalyseurs dans les mobilisations identitaires des populations musulmanes, comme dans la négociation de leurs modes d'insertion au sein des sociétés d'accueil47. Or, les musulmans d'origine balkanique sont restés en retrait de ces mobilisations, et ce pour plusieurs raisons. D'une part, ces diaspo-

\footnotetext{
44 Sur les conversions religieuses de certains Albanais du Kosovo, en partículier après le 11 septembre 2001, voir Kostovicova (Denisa), Prestreshi (Albert), art.cit., pp. 1092-1093.

45 Colic-Peiskex (Val), art.cit., pp. 626,628 .

46 Voir également note 36 .

47 Voir entre autres Kepel (Gilles), A l'ouest d'Allah, Paris : Seuil, 1994 ; Dassetto (Felice), ed., op.cit. ; Metcalf (Barbara), ed., Making Muslim Space in North America an Europe, Berkeley : University of California Press, 1996 ; Vertovec (Steven), Peach (Ceri), Islam in Europe. The Politics of Religion and Community, Basingstoke : Macmillan, 1997 ; Haddad (Yvonne), Smith (Jane I.), eds., Muslim Minorities in the West : Visible and Invisible, Walnut Creek : Altamira Press, 2002.
} 
ras sont récentes, et restent de taille modeste en comparaison d'autres communautés telles que la diaspora turque en Allemagne et en Scandinavie ou les diasporas turque et marocaine en Belgique. Même lorsque certains représentants des diasporas musulmanes balkaniques participent, au niveau local ou national, à des fédérations religieuses pluriethniques ou à des instances représentatives du culte musulman, ils n'y occupent donc qu'une place marginale. D'autre part, pour les musulmans balkaniques, l'expérience d'insertion minoritaire au sein d'une société sécularisée précède la migration, comme cela a déjà été mentionné plus haut.

Certes, dans les Balkans également, le réveil politique national des musulmans balkaniques va de pair avec la mise en avant de certains marqueurs identitaires d'origine religieuse, et donc avec une visibilité accrue de l'islam ${ }^{4}$. Mais ce processus ne se retrouve pas en contexte diasporique : dans ce dernier cas, en effet, la priorité accordée à la cause nationale amène les musulmans d'origine balkanique à écarter toute manifestation ou revendication religieuse qui pourrait les compromettre aux yeux des autorités ou des opinions publiques des pays d'accueil. De ce point de vue, les diasporas musulmanes balkaniques se distinguent non seulement des autres populations musulmanes de l'UE, mais aussi de leurs propres communautés de référence. Enfin, les musulmans balkaniques vivant en contexte "semi-diasporique" semblent constituer des cas à part, dans lesquels les liens entre mobilisation identitaire et visibilité religieuse se complexifient d'avantage, comme l'attestent dans plusieurs centres urbains des Balkans (Athènes, Belgrade 49 , Zagreb, Rijeka, Ljubljana, etc.) l'émergence de revendications liées à la pratique du culte musulman (ouverture d'une mosquée centrale, création de parcelles musulmanes dans les cimetières de la ville, etc.), et rappelant celles existant en Europe occidentale, ou encore la tentative des animateurs de l'association Filotita de s'appuyer sur la masse des musulmans étrangers pour s'ériger en partenaire légitime des autorités grecques.

\section{« ANCIENS » ET « NOUVEAUX » MUSULMANS D'EUROPE}

Lorsque les musulmans des Balkans parlent d'“islam européen", c'est le plus souvent au sens d'un "islam autochtone" dont ils excluent sans état d'âme les populations musulmanes arrivées en Europe après la Seconde Guerre mon-

$4^{8}$ Voir Bougarel (Xavier), Clayer (Nathalie), éds., op.cit.

49 Sur la situation de la communauté musulmane à Belgrade, voir Tanasković (Darko), " Islam u Beogradu "(L'islam à Belgrade), in U dijalogu s Islamom (En dialogue avec l'Islam), Gornji Milanovac : Dečje novine, 1992. 
diale ${ }^{50}$. À l'inverse, dans les débats actuels sur l'émergence d'un "islam d'Europe" (ou "Euro-Islam"), à savoir de pratiques et d'interprétations de la religion musulmane adaptées au contexte des sociétés européennes, les musulmans balkaniques ne sont généralement pas pris en compte, dans la mesure où ils ne sont pas (encore) citoyens de l'Union européenne ${ }^{51}$. La période de la guerre en Bosnie-Herzégovine n'a, de ce point de vue, constitue qu'une parenthèse stérile : le destin tragique de la communauté bosniaque a certes mobilisé les opinions publiques occidentales et musulmanes, et Alija Izetbegović a été érigé en modèle de "musulman européen", mais c'est au prix du passage sous silence des clivages et des évolutions internes à l'islam bosniaque ${ }^{52}$.

Il faut donc se demander quelle peut être l'articulation présente et future de ces deux acceptions divergentes de $\mathrm{l}^{\prime \prime}$ islam européen", et quelle place y occupent les musulmans d'origine balkanique vivant en Europe occidentale. Par un paradoxe apparent, ces derniers ont surtout joué un rôle crucial dans l'implantation de l'islam en Europe occidentale et dans les contacts entre communautés musulmanes des Balkans et acteurs religieux du monde musulman avant 1990 . Ainsi, dans les années 1960 et 1970 , la petite communauté albanaise de Belgique a joué un rôle actif dans l'ouverture du premier Centre islamique à Bruxelles 53 , et des émigrés politiques bosniaques tels que Smail Balić, Salem Hadžić ou Teufik Velagić ont joué un rôle central dans la vie de la petite communauté islamique autrichienne ${ }^{54}$. À l'inverse, dans les années 1970 et 1980 , certains Gastarbeiter bosniaques et albanais ont fréquenté des mosquées turques ou arabes avant d'initier la création des premiers jama'ats yougoslaves. Dès cette époque, toutefois, la croissance numérique rapide des différentes populations musulmanes présentes en Europe occidentale et l'émergence de nouveaux acteurs associatifs ont entrainé une plus forte compartimentation ethno-nationale de la vie religieuse, et une marginalisation des musulmans balkaniques arrivés au lendemain de la Seconde Guerre mondiale. Jusqu'en 1990, de nombreux échanges entre communautés musulmanes des Balkans et

50 Au milieu des annees 1970, l'anthropologue amèricain William Lockwood pouvait encore intituler "Musulmans européens" son livre pionnier sur les Musulmans de Bosnie-Herzégovine. Voir Lockwood (William), European Moslems : Economy and Ethnicity in Western Bosnia, New York : Academic Press, 1975.

${ }^{51}$ Voir par exernple Roy (Olivier), Vers un Islam europeen (op.cit.) ; Nielsen (Jorgen), op.cit.. Pour une tentative de couvrir l'ensemble des populations musulmanes d'Europe, voir Nonneman (Gerd), Niblock (Tim), Szajkowski (Bogdan), eds., Mustim Communities in the New Europe, London : Ithaca Press, 1996 ; Dassetto (Felice), Marechal (Brigitte), Nielsen (Jorgen), eds., op.cit.

52 Voir Bougarel (Xavier), "L'islam et la guerre en Bosnie-Herzégovine : l'impossible dèbat ? ", L'Autre Europe, (36-37), hiver 1998/1999 : Bougarel (Xavier). " Trois définitions de l'islam en Bosnie-Herzégovine ", Archives des sciences sociales des religions, (115), juillet-septembre 2001 (dossier "Islam et politique dans le monde ex-communiste ").

53 Voir Sula (Selvi), art.cit.

54 Voir Karié (Enes), Filandra (Šaćir), op.cit. 
acteurs religieux du monde musulman se sont réalisés par le biais de l'Europe occidentale, comme l'attestent les contacts entre Gastarbeiter bosniaques et albanais et certains réseaux transnationaux de type confrérique ou militant, l'importation" de nouveaux modèles idéologiques ou architecturaux, ou encore le rôle joué en 1983 par Teufik Velagić dans le séjour en Iran d'une délégation d'islamistes bosniaques. Mais ce "détour diasporique" a perdu une bonne partie de son importance après 1990 , lorsque l'effondrement des régimes communistes et l'ouverture des frontières ont permis aux acteurs religieux du monde musulman de s'implanter directement dans les Balkans.

Quinze ans après la disparition du Rideau de fer, et alors même que les discussions sur l'élargissement de l'UE et l'émergence d'un "islam d'Europe" s'intensifient, les diasporas musulmanes balkaniques semblent repliées sur leur identité ethno-nationale d'une part, sur un "islam européen" dont elles revendiquent le monopole d'autre part. Là encore, cette situation reflète largement celle qui prévaut au sein de leurs communautés de référence. Après tout, les violences et les discriminations dont sont victimes les Roms, les Bosniaques et les Goranis au Kosovo montrent que les clivages ethniques divisent aussi les populations musulmanes dans les Balkans, et les institutions religieuses islamiques des pays balkaniques ont généralement plus de liens avec la Direction des affaires religieuses (Diyanet) de Turquie qu'avec les forums islamiques paneuropéens qui se sont développés ces dernières années. Le cas particulier de Mustafa Cerić, Reis-ul-Ulema de Bosnie-Herzégovine, montre toutefois que les choses ne sont ni aussi simples, ni aussi figées qu'il ne semble à première vue.

Parmi les principaux oulémas des Balkans, Mustafa Cerić est le seul à avoir une expérience diasporique importante : avant de devenir Reis-ul-Ulema en 1993, en effet, il a été imam du Centre islamique bosniaque de Chicago de 1981 à 1986, puis imam principal de la mosquée de Zagreb de 1986 à 1993. Il est le seul à participer au Conseil européen pour les fatwas et la recherche créé en $1997^{55}$, et à insister régulièrement sur la nécessité de doter la religion musulmane d'instances représentatives à l'échelle européenne, ou de structures de formation pour imams situeses en Europe. Pourtant, cette vocation "paneuropéenne" de Mustafa Cerić - que lui reprochent du reste certains oulémas bosniaques - ne se retrouve pas dans la diaspora bosniaque, les jama'ats bosniaques et leurs fédérations nationales - telles que l'Union des paroisses islamiques des Bosniaques en Allemagne (Vereinigung islamischer Gemeinden der Bosniaker) - s'impliquant peu dans les forums pluriethniques ou les in-

55 Voir Caeiro (Alexandre), "Transnational 'Ulama, European Fatwas, and Islamic Authority : A Case Study of the European Council for Fatwa and Research ", in Van Bruinessen (Martin), Allievi (Stefano), eds., Production and Dissemination of Islamic Knowledge in Western Europe, London : Routledge (forthcoming) ; Caeiro (Alexandre), "Pan-European Fatwas and National Contexts : Dilemmas of Ifta' in Western and Eastern Europe ", papier présenté lors de la conférence Les musulmans balkaniques et l'islam en Europe de l'Ouest, Poitiers, 13-14 novembre 2003. 
stances représentatives du culte musulman. Il s'agit là moins d'un écart entre diaspora et communauté de référence que d'une divergence entre des stratégies d'affirmation institutionnelle et des stratégies locales d'intégration. Mais cette divergence se traduit bel et bien par des définitions différentes du lien entre identité religieuse et identité nationale, et par des positionnements différents dans l'espace public : à des institutions religieuses souhaitant s'ériger en médiateurs entre les sociétés européennes et les "nouveaux musulmans" européens (en cela, les choix stratégiques de Mustafa Cerić rappellent, à une autre échelle, ceux des animateurs de Filotita) s'opposent une majorité de musulmans balkaniques, qui refusent ce rôle de médiation perçu comme une menace pour leur cohésion nationale et leur intégration sociale, et une minorité de jeunes musulmans, qui s'identifient à un islam radical et anti-occidental.

À court terme, les diasporas musulmanes balkaniques ne constituent sans doute pas un facteur décisif dans le rapprochement entre populations musulmanes de l'Europe occidentale et de l'Europe balkanique, ou dans l'élaboration d'un "islam d'Europe". D'autres facteurs jouent sans doute un rôle plus déterminant, tels que le recours commun à certaines institutions européennes (Cour européenne des droits de l'Homme, Parlement européen, etc.) ou la traduction d'auteurs s'intéressant à la question de l'islam en Europe, tels que Yusuf al-Qaradawi ou Tariq Ramadan. Toutefois, l'intensification des échanges entre musulmans de l'Europe occidentale et de l'Europe balkanique et les efforts de définition d'un "islam d'Europe" ne peuvent que favoriser la redécouverte de l'expérience minoritaire des musulmans balkaniques, et de l'héritage institutionnel et intellectuel qui lui est lié56. Par ailleurs, l'élargissement de l'UE aux pays balkaniques et la transformation de certains d'entre eux en terres d'immigration - dans un pays comme la Roumanie, le nombre de musulmans étrangers dépasse désormais celui des musulmans turcophones de la Dobroudja 57 - brouillent peu à peu la frontière entre musulmans "autochtones" et "allogènes", islam "européen" et "non-européen". Qu'ils le veuillent ou non, les musulmans d'origine balkanique vivant dans l'UE contribuent eux aussi à ce brouillage. Or, à plus ou moins long terme, ces processus lents et complexes ne peuvent qu'influencer la manière dont se positionnent les diasporas musulmanes balkaniques, et différents acteurs politiques ou religieux en leur sein.

56 Voir entre autres Karčić (Fikret), The Bosniaks and the Challenges of Modernity. Late Ottoman and Habsburg Times, Sarajevo : el-Kalem, 1999 ; Karić (Enes), " Islamic Thought in Bosnia-Herzegovina in the $20^{\text {th }}$ Century. Debates on Revival and Reform n, Islamic Studies, 41 (2), 2002.

57 Voir Lederex (György), Countering Islamist Radicals in Eastern Europe, Camberley : Conflict Studies Research Center, 2005, p. 8, accessible sur 〈http://www.da.mod.uk/CSRC〉. 